\title{
Susceptibility Weighted Imaging: A Novel Method to Determine the Etiology of Aqueduct Stenosis
}

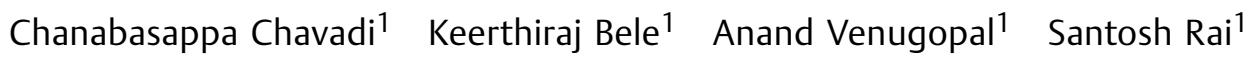 \\ ${ }^{1}$ Department of Radiodiagnosis, Kasturba Medical College, Manipal \\ Address for correspondence Chanabasappa Chavadi, DNB, Flat No. C- \\ University, Mangalore, India \\ 1-13, 3rd Floor, K.M.C Staff Quarters, Light House Hill Road, \\ Mangalore 575001, India (e-mail: chavadidoc@gmail.com). \\ Indian Journal of Neurosurgery 2016;5:44-46.
}

\begin{abstract}
The stenosis of aqueduct of Sylvius (AS) is a very common cause of obstruction to cerebrospinal fluid. Multiple etiologies are proposed for this condition. Because treatment is specific for correctable disorder, assessment of etiology gains importance. A case of pediatric hydrocephalus was diagnosed with stenosis of AS on magnetic resonance imaging (MRI). Susceptibility-weighted imaging (SWI) demonstrated blooming in the distal aqueduct and lateral ventricle, which was not

Keywords

- susceptibility weighted imaging

- aqueductal stenosis

- magnetic resonance imaging

- hydrocephalus seen on routine MRI sequences. The findings suggest that old hemorrhage is a cause of chemical arachnoiditis and adhesions causing aqueduct stenosis and hydrocephalus. To our knowledge literature is very scarce, wherein SWI is being used to confirm blood products as a cause of aqueduct stenosis; hence SWI should be routine protocol in imaging of pediatric hydrocephalus. Etiology, clinical presentation, role of imaging, and, in particular, SWI in evaluation of aqueductal stenosis is discussed.
\end{abstract}

\section{Introduction}

Aqueduct of Sylvius (AS) is the narrowest segment of the cerebrospinal fluid (CSF) pathway and is the most common site of intraventricular obstruction to the flow of CSF. Stenosis of the AS is responsible for 6 to $66 \%$ of cases of hydrocephalus in children and 5 to $49 \%$ in adults. ${ }^{1,2}$ There are two peaks of distribution for age: one in the first year of life, the other in the adolescence. Susceptibility-weighted imaging (SWI) is a unique gradient-echo magnetic resonance imaging (MRI), which is flow-compensated and uses magnitude and phase information. Phase postprocessing accentuates the magnetic properties of extravascular blood products and produces high contrast, when compared with other conventional MRI sequences. ${ }^{3}$ SWI is being extensively used in trauma, vascular pathologies and tumors causing microbleeds, multiple sclerosis, and stroke. In our case, SWI confirmed the cause of stenosis of AS and the case is being discussed. Etiology, pathogenesis, and imaging of aqueduct stenosis are summarized.

received

April 8, 2015

accepted

November 18, 2015

published online

February 19, 2016 10.1055/s-0036-1572367. ISSN 2277-954X.

\section{Case Report}

An 8-month-old child presented with increased head size, developmental delay, and an episode of seizure. No history of birth asphyxia was found. Laboratory investigations were normal. MRI revealed enlargement of the lateral and third ventricle ( - Fig. 1a, b) and dilation of proximal aqueduct with narrowing at the distal segment secondary to adhesions (- Fig. 1c, d). The temporal horn diameter was $28 \mathrm{~mm}$ and ventricle size index (VSI) was $50 \%$ suggestive of significant hydrocephalus. There was narrow angle of the frontal horn and effacement of the sulci at vertex ( $\mathbf{- F i g}$. 1b). SWI demonstrated blooming at the distal AS (-Fig. 1f, g), confirming the etiology as adhesions due to extravascular blood products. Blooming due to hemorrhage was also noted occipital horn (-Fig. 1h) with dilation of the suprapineal recess and absence of normal flow void in the fourth ventricle (-Fig. 1e).

\footnotetext{
(c) 2016 Neurological Surgeons' Society of India
}

License terms 

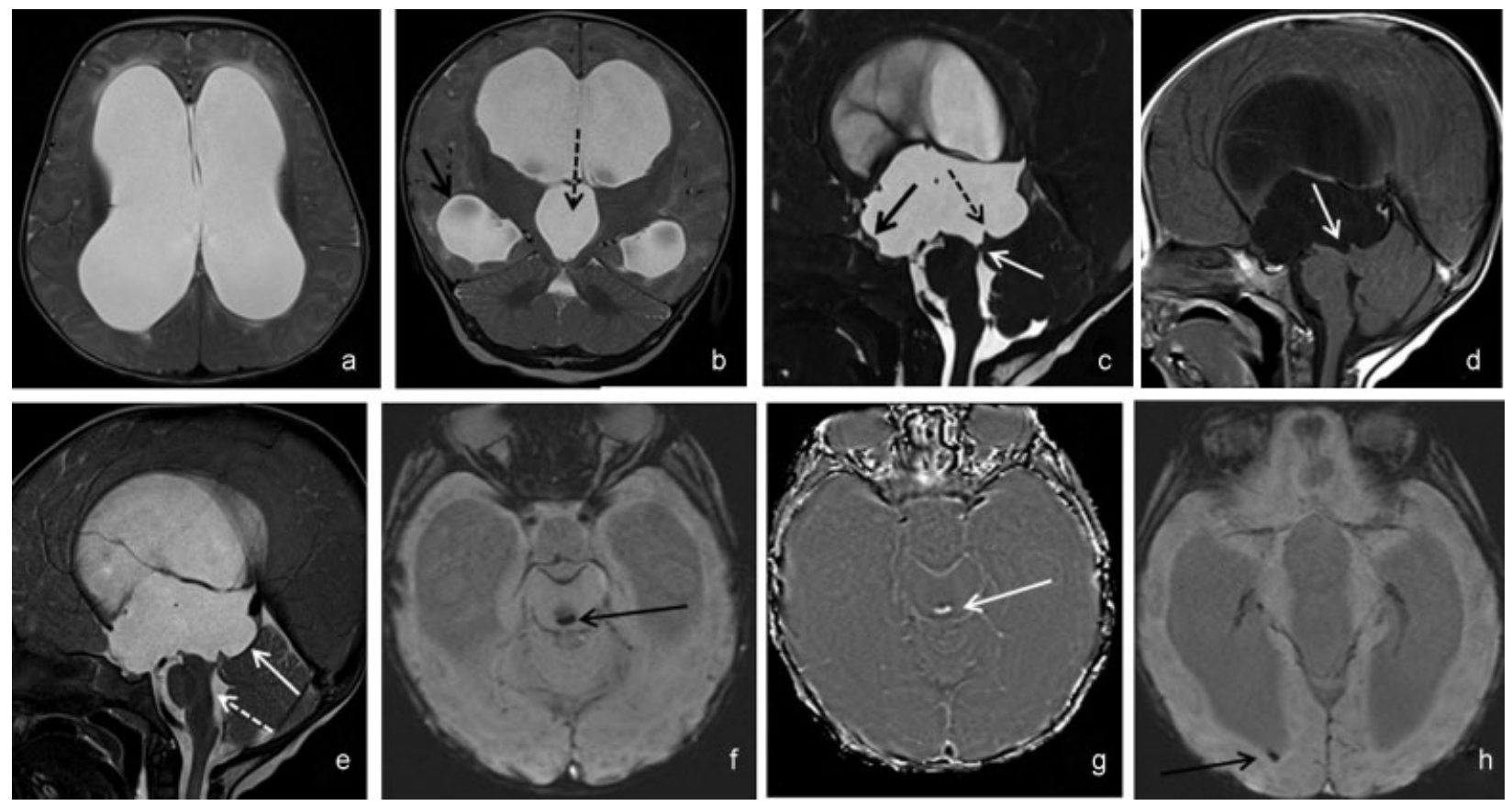

Fig. 1 (a) Axial T2: enlargement of lateral ventricle; (b) coronal T2: dilated temporal horn (black arrow) and third ventricle (interrupted black arrow); (c) sagittal CISS: stenosis in the distal aqueduct (white arrow) and posterior rotation of the tectal plate (interrupted black arrow), thinning of the floor of the third ventricle involving the chiasm and lamina terminalis (black arrow); (d) sagittal postcontrast T1: flaring of proximal aqueduct duct (white arrow), no abnormal enhancement in region of aqueduct; (e) sagittal T2: posterior bulging of the suprapineal recess (white arrow) and absence of normal flow void in the fourth ventricle (interrupted white arrow); ( $\mathbf{f}$ and $\mathbf{g}$ ) axial SWI in magnitude and phase images, respectively, demonstrated blooming in the distal aqueduct of Sylvius (arrow) suggestive of extravascular blood products; (h) axial SWI in magnitude image: blooming in occipital horn suggestive of intraventricular hemorrhage (black arrow).

\section{Discussion}

The AS is a tubular channel situated in the dorsal midbrain, connecting the third and the fourth ventricles. Stenosis of AS may be tumoral or may be nontumoral which is referred to as intrinsic aqueductal stenosis. In about three-quarters of patients with aqueductal stenosis, the etiology of the disorder is not known. ${ }^{1}$ In the remaining cases, it can be attributed to different causes, namely genetic, infectioninflammation, prematurity-related intraventricular hemorrhage, in which case aqueductal stenosis may develop in the acute phase, secondary to direct obstruction of aqueduct by blood, or in a chronic phase, secondary to organization of clots. ${ }^{4}$ Other causes include intoxications and deficiencies, congenital malformations, and vascular malformations. Russell ${ }^{5}$ classified nontumoral aqueduct stenosis by histopathologic studies into four subtypes: (1) stenosis without gliosis of the surrounding tissue, proposed to be due to "developmental errors"; (2) forking caused by incomplete fusion of the median fissure; (3) membranous obstruction; (4) gliosis usually a reaction to irritant agents, such as hemorrhage, infection, or toxic agents, and is often part of a widespread ependymitis of the ventricles.

In the first year of life, the clinical presentation usually consists of rapid enlargement of the circumference of the head. $^{3}$ Other features include retarded psychomotor development, cognitive dysfunction, temporary headache, urinary incontinence, seizures, and growth retardation. Sometimes, decompensation may follow minor head injury, febrile affections, or subarachnoid hemorrhage. ${ }^{3}$ Visual disturbances are related to papilledema, and chronic compression of the optic chiasm is exerted by an enlarged third ventricle. Endocrine manifestations occur in $10 \%$ of adolescents and adults, due to chronic compression of the hypothalamo-hypophyseal axis by the enlarged anterior third ventricle.

Imaging is the cornerstone for diagnosis, intrauterine diagnosis by ultrasonography primarily by excluding other causes of hydrocephalus in presence of triventriculomegaly with macrocephaly. Antenatal MRI may allow diagnosis of aqueductal stenosis by showing hydrocephalus in association with the normal fourth ventricle and absence of aqueductal lumen. ${ }^{6}$ MRI is investigation of choice for evaluation of hydrocephalus and aqueductal stenosis. Anatomical deformations of bulging of the third ventricle are better evaluated. The optimal view for the evaluation of anatomical details is a midline sagittal section. ${ }^{6}$ On $\mathrm{T} 1$ weighted image (WI) sequences, the aqueduct has the same signal intensity as CSF, whereas on T2 WI, a hypointense signal is seen as a result of CSF flow. This "flow void" extending inferiorly into the superior aspect of the fourth ventricle has been considered as an important sign for diagnosis of patency of aqueduct, ${ }^{7}$ with a few limitations. If the aqueduct remains visible, the obstruction is 
presumably caused by a membrane. Ultra-thin threedimensional constructive steady-state sequences (CISS) sequence due to inherent high CSF-to-brain contrast provides exquisite detail of the ventricular system and permits better identification of aqueductal obstruction by membranes, webs, hypoplasia or cysts, and tumors. ${ }^{8}$ The absence of CSF flow at the aqueductal level in flowsensitive phase contrast MRI is a reliable, reproducible, and rapid method, which supports the diagnosis of stenosis of AS in patients with clinical and/or radiologic suggestion of obstructive hydrocephalus. ${ }^{9}$

In cases of long-standing hydrocephalus, CSF pulsations against the thinnest segment of the ventricular walls may determine focal enlargement of some portions of the ventricular system. MRI is helpful by detecting and correctly explaining pulsion diverticula, third ventricle bulging in the chiasmatic and interpeduncular cisterns, and cystic expansion of the suprapineal recess, subependymal dissection, and spontaneous ventriculocisternostomies. ${ }^{10}$

\section{Conclusion}

Triventriculomegaly is usually accepted as a sign of stenosis of AS. The etiology of stenosis of AS may be obscure on routine MRI. Our observation suggests that detection of blooming on SWI in distal aqueduct with flaring of the proximal aqueduct confirms the causative factor of stenosis of AS, being secondary to intraventricular hemorrhage. To our knowledge literature is very scarce, wherein SWI is being used to confirm blood products as a cause of aqueduct stenosis. With varied etiology and limitation of routine MRI sequence, SWI is a novel method, and should be a part of routine MRI protocol for imaging in congenital and pediatric hydrocephalus.

\section{Conflict of Interest}

The authors declare that there is no actual or potential conflict of interest in relation to this article. The manuscript has been read and approved by all the authors, the requirements for authorship as stated earlier in this document have been met, and that each author believes that the manuscript represents honest work.

\section{Source(s) of Support}

None.

Acknowledgment

None.

\section{References}

1 Cinalli G, Spennato P, Nastro A, et al. Hydrocephalus in aqueductal stenosis. Childs Nerv Syst 2011;27(10):1621-1642

2 Robertson IJ, Leggate JRS, Miller JD, Steers AJ. Aqueduct stenosis-presentation and prognosis. Br J Neurosurg 1990; 4(2):101-106

3 Haacke EM, Xu Y, Cheng YC, Reichenbach JR. Susceptibility weighted imaging (SWI). Magn Reson Med 2004;52(3): 612-618

4 Boop FA. Posthemorrhagic hydrocephalus of prematurity. In: Cinalli C, Maixner WJ, Sainte-Rose C, eds. Pediatric Hydrocephalus. Milan, Germany: Springer; 2004:121-131

5 Russell DS. Observations on the pathology of hydrocephalus. Medical Research Council Special Report Series No. 265. London, UK: His Majesty's Stationery Office; 1949

6 Brunelle F. Modern imaging of hydrocephalus. In: Cinalli C, Maixner WJ, Sainte-Rose C eds. Pediatric Hydrocephalus. Milan, Germany: Springer; 2004:79-93

7 Citrin CM, Sherman JL, Gangarosa RE, Scanlon D. Physiology of the CSF flow-void sign: modification by cardiac gating. AJNR Am J Neuroradiol 1986;7:1021-1024

8 Laitt RD, Mallucci CL, Jaspan T, McConachie NS, Vloeberghs M, Punt J. Constructive interference in steady-state 3D Fouriertransform MRI in the management of hydrocephalus and third ventriculostomy. Neuroradiology 1999;41(2):117-123

9 Stoquart-El Sankari S, Lehmann P, Gondry-Jouet C, et al. Phasecontrast MR imaging support for the diagnosis of aqueductal stenosis. AJNR Am J Neuroradiol 2009;30(1):209-214

10 Lapras C, Bret P, Patet JD, Huppert J, Honorato D. Hydrocephalus and aqueduct stenosis. Direct surgical treatment by interventriculostomy (aqueduct canulation). J Neurosurg Sci 1986;30(1-2):47-53 